\title{
Epidemiologia da AIDS no Brasil, região Centro-Oeste e Distrito Federal, 2008-2018
}

\author{
Epidemiology of AIDS Brazil, Central-West region and Distrito Federal, 2008-2018 \\ Epidemiología del SIDA en Brasil, región Centro-Oeste y Distrito Federal, 2008-2018
}

Recebido: 21/04/2021 | Revisado: 30/04/2021 | Aceito: 03/05/2021 | Publicado: 17/05/2021

\author{
Maria Paula Meireles Fenelon \\ ORCID: https://orcid.org/0000-0002-8610-3881 \\ Centro Universitário de Brasília, Brasil \\ E-mail: mariapfenelon@gmail.com \\ Maria Clara Potiguara Azevedo Teixeira \\ ORCID: https://orcid.org/0000-0003-1405-1979 \\ Centro Universitário de Brasília, Brasil \\ E-mail: mclaraatx@gmail.com \\ Mylena Valadares Silva \\ ORCID: https://orcid.org/0000-0002-8610-3881 \\ Centro Universitário de Brasília, Brasil \\ E-mail: valadaresmsilva@gmail.com \\ Bruna de Paula Gonçalves Sousa Lyra \\ ORCID: https://orcid.org/0000-0003-1918-1027 \\ Centro Universitário de Brasília, Brasil \\ E-mail: brunalyra98@gmail.com \\ Lara Medeiros Amaral \\ ORCID: https://orcid.org/0000-0002-4451-8691 \\ Universidade Católica de Brasília, Brasil \\ E-mail: amaral.m.lara@gmail.com \\ Gerson Fernando Mendes Pereira \\ ORCID: https://orcid.org/0000-0001-8886-1662 \\ Secretaria de Vigilância em Saúde, Brasil \\ E-mail: gerson.pereira@AIDS.gov.br
}

\begin{abstract}
Resumo
Objetivo: Analisar a situação epidemiológica do HIV/AIDS no Brasil, na região Centro-Oeste (CO) e Distrito Federal (DF) entre 2008 e 2018. Métodos: Estudo de caráter quantitativo, observacional e descritivo com dados do Boletim Epidemiológico HIV/AIDS 2019, do Ministério da Saúde, referente aos casos e óbitos por HIV/AIDS entre 2008 e 2018. Resultados: Nos últimos dez anos, a taxa de detecção de AIDS declinou no Brasil (17,9\%), no CO $(4,4 \%)$ e no DF (25,8\%). Nesse período, o Brasil passou de 6,2 para 5,3 óbitos/100.000 habitantes e, no CO, de 5,3 para 4,7 óbitos/100.000 hab. Em todo o período, o DF apresentou coeficiente de mortalidade inferior ao nacional, com menor taxa em 2018 (3,6/100.000 hab.). Conclusão: Observa-se queda da taxa de detecção de AIDS e do coeficiente de mortalidade no Brasil, CO e DF.
\end{abstract}

Palavras-chave: AIDS; SUS; Carga viral; DST; Infecções por HIV.

\begin{abstract}
Objective: To report and describe the epidemiological situation of HIV / AIDS in Brazil, in the Central West (CW) region and in the Distrito Federal (DF) between 2008 and 2018. Methods: Observational and descriptive study using data from the HIV/AIDS 2019 Epidemiological Bulletin, from the Ministry of Health, referring to HIV/AIDS cases and deaths between 2008 and 2018. Results: In the last 10 years, the AIDS detection rate has fallen in Brazil (17.9\%), in CW (4.4\%) and in DF (25.8\%). During this period, Brazil went from 6.2 to 5.3 deaths/100,000 inhabitants and in the CW from 5.3 to 4.7 deaths/100,000 inhab. In the whole period, the DF presented a mortality rate lower than the national, having showed the lowest rate (3.6/100,000 inhab.) in 2018. Conclusion: Decreases in the rate of AIDS detection and mortality coefficient are observed in Brazil, CW and DF.
\end{abstract}

Keywords: AIDS; SUS; Viral load; STD; HIV infections.

\section{Resumen}

Objetivo: Analizar la situación epidemiológica del VIH/SIDA en Brasil, Centro Oeste brasileño (CO) y Distrito Federal (DF) entre 2008 y 2018. Métodos: Estudio observacional y descriptivo utilizando datos del Boletín Epidemiológico de VIH/SIDA 2019, del Ministerio de Salud, refiriéndose a los casos y muertes por VIH/SIDA entre 2008 y 2018. Resultados: En los últimos 10 años, la tasa de detección de SIDA ha disminuido en Brasil (17.9\%), en el CO (4.4\%) y en el DF (25,8\%). Durante este período, Brasil pasó de 6.2 a 5.3 muertes/100,000 habitantes. En el CO de 5.3 a 4.7 muertes/100,000 hab. En todo el período, el DF presentó una tasa de mortalidad más baja que la nacional, 
en 2018 mostró la tasa más baja (3.6/100,000 habitantes). Conclusión: Se observa una disminución en la tasa de detección de SIDA y el coeficiente de mortalidad en Brasil, CO y DF.

Palabras clave: SIDA; SUS; Carga viral; ETS; Infecciones por VIH.

\section{Introdução}

\section{A história do HIV no mundo}

A história da epidemia de HIV/AIDS tem início entre 1977 e 1978 quando foram descobertos os primeiros casos nos EUA e no Haiti. Quatro anos depois, na África Central, essa nova doença foi classificada como Síndrome da Imunodeficiência Adquirida, a AIDS (Acquired Immunodeficiency Syndrome, em inglês). No mesmo ano, o nome "Doença dos 5H" se tornou popular, sendo que cada letra representava um grupo populacional: homossexuais, hemofílicos, haitianos, heroinômanos (usuários de heroína injetável) e hookers (denominação em inglês para as profissionais do sexo). Em 1985, o primeiro teste anti-HIV foi disponibilizado para diagnóstico e descobriu-se que a AIDS, propriamente dita, é a fase final da doença causada por um retrovírus, denominado Vírus da Imunodeficiência humana (Human Immunodeficiency Virus, em inglês). Em 1987 tem início a utilização do AZT (Zidovudina), o primeiro medicamento que reduziu a multiplicação do HIV. (Ministério da Saúde, 2020)

\section{A história do HIV no Brasil}

A epidemia de AIDS teve início no Brasil na década de 1980, sendo a maioria dos casos concentrados em São Paulo. A doença chegou a ser chamada de "praga gay", pois a maioria dos acometidos eram homossexuais, sendo que o primeiro caso de AIDS no sexo feminino no país foi registrado apenas em 1983 (Ministério da Saúde, 2020).

No início da década, ainda não havia diagnóstico laboratorial específico para o HIV, o que contribuía para que muitos chegassem a negar sua existência (Barros, 2016). Com o avançar do tempo, iniciaram-se medidas governamentais para controle da doença, e em fevereiro de 1985 foi criado o Programa Nacional de Doenças Sexualmente Transmissíveis (DST) e AIDS (PN-DST AIDS), passando-se a realizar vigilância epidemiológica, educação e informação em saúde, testagens em massa e caracterização da epidemia como suscetível de afetar toda a população, no intuito de reduzir o estigma e discriminação sobre os grupos mais afetados (Barros, 2016). Em 1987, os Ministérios da Saúde e do Trabalho incluíram as DST/AIDS na Semana Interna de Prevenção de Acidentes no Trabalho e Saúde (Ministério da Saúde, 2020).

O ano de 1991 é marcado pelo início do processo para a aquisição e distribuição gratuita de antirretrovirais no Brasil (Ministério da Saúde, 2020). Além de o tratamento oferecer melhora à saúde e ao bem-estar dos portadores da doença, há interrupção da transmissão do HIV, impactando diretamente na redução do número de novos casos e óbitos (Vella, 2012). Observou-se então queda da mortalidade no país a partir de 1996, quando foi introduzido o tratamento pelo serviço público de saúde.

\section{O panorama atual do HIV/AIDS}

Apesar dos avanços descritos, o número alarmante de novas infecções por HIV demonstra sua relevância como um problema de saúde pública (Levy, 2012). Em 2015 foram relatadas 2,1 milhões de novas infecções por HIV ao redor do mundo, contabilizando um total de 36,7 milhões de pessoas vivendo com essa doença (Reis, 2007).

Fatores como baixa percepção de risco, estigma, preconceito, redução da testagem em grupos de risco, má adesão à terapia antirretroviral (TARV) e até mesmo maior abrangência e capacidade de notificação podem ter contribuído para esse número alarmante de novos casos (Guimarães, 2017). Em relação à TARV, apenas $46 \%$ dos pacientes diagnosticados com HIV no mundo em 2015 estavam em uso da terapia (UNAIDS, 2016). 
O HIV ainda demonstra importante morbimortalidade na atualidade. As causas responsáveis são múltiplas, de tal maneira que as infecções oportunistas e neoplasias, apesar de majoritárias, não dominam esse cenário. São consideradas causas importantes a insuficiência hepática, em geral associada a infecções pelo vírus da hepatite C (HCV), coinfecção com a tuberculose e as complicações associadas aos efeitos adversos dos antir-retrovirais (Pereira, 2007).

A epidemia do HIV no Brasil, segundo a Organização Mundial da Saúde (OMS), ainda se configura como concentrada: as prevalências em populações-chave (usuários de drogas injetáveis, homens que fazem sexo com homens e mulheres profissionais do sexo) são mais altas do que na população em geral (Pereira, 2018).

Em comparação com outros programas de controle de doenças, observa-se que o Brasil continua a combater a AIDS, na defesa intransigente dos direitos humanos, a privilegiar a produção local e distribuição gratuita de preservativos e antirretrovirais (ARV), a promover a implantação da rede pública de laboratórios e de serviços para cuidar das pessoas vivendo com AIDS e a destinar financiamento para pesquisas. Nesse contexto, verificam-se ainda muitos desafios para a sustentabilidade do programa brasileiro, sendo o maior deles o enfrentamento da disparidade, da pobreza e da discriminação, fatores que aumentam a vulnerabilidade das pessoas em relação ao HIV/AIDS e dificultam o acesso à necessária e adequada prevenção, aos cuidados médicos e a adesão ao tratamento. (Greco, 2016)

Para diminuir os desafios no combate ao HIV/AIDS, é necessário conhecer as características do país, dos seus estados e dos seus municípios, de forma a instituir programas personalizados e específicos para as diversas realidades epidemiológicas, sociais e econômicas do Brasil. Ao traçar um panorama dos casos e óbitos é possível relatar diferenças operacionais importantes, que devem ser consideradas de forma a sugestionar estratégias de saúde pública específicas para cada localidade.

Dessa forma, o presente estudo objetiva descrever a situação epidemiológica do HIV/AIDS no Brasil, no CentroOeste e no Distrito Federal nos anos de 2008 a 2018. Para isso, serão analisadas a taxa de detecção de AIDS e o coeficiente de mortalidade no Brasil, Centro-Oeste e Distrito Federal.

\section{Metodologia}

Trata-se de um estudo de caráter quantitativo, observacional e descritivo, de base populacional e sem coletas de material biológico. A base metodológica para a pesquisa foi fornecida por Koche (2011), que descreve a pesquisa descritiva, ou não experimental, como um estudo de relações entre duas ou mais variáveis de um dado fenômeno sem manipulá-las a priori, sendo feita apenas a constatação de sua manifestação a posteriori. Os dados foram obtidos a partir do Boletim Epidemiológico do Ministério da Saúde, referente aos casos de AIDS no Brasil, na região Centro-Oeste (CO) e no Distrito Federal (DF), assim como os óbitos, no período de 2008 a 2018. A busca de informações para fundamentar o presente estudo foi feita nas bases de dados Google Acadêmico e Scielo, utilizando como buscadores os termos: AIDS, SUS, carga viral, DST e infecções por HIV. Foram utilizados artigos em inglês e português.

As fontes dos dados secundários utilizadas foram: (i) Sistema de Informação de Agravos de Notificação (Sinan); (ii) Sistema de Informações sobre Mortalidade (SIM); (iii) Sistema de Controle de Exames Laboratoriais da Rede Nacional de Contagem de Linfócitos T-CD4+/CD8+ e Carga Viral do HIV (Siscel); e (iv) Sistema de Controle Logístico de Medicamentos (Siclom). Esses sistemas de informação são utilizados pelo Ministério da Saúde para ampliar e habilitar os dados de notificação de AIDS em crianças e adultos.

- O Sinan é alimentado, principalmente, pela notificação e investigação de casos de doenças e agravos que constam da lista nacional de doenças de notificação compulsória (Laguardia, 2004).

- O SIM foi criado para a obtenção regular de dados sobre mortalidade no país (Morais, 2017).

- O Siscel é um sistema web para exames de carga viral do HIV, contagem de linfócitos T-CD4+/CD8+ e genotipagem do HIV na rede pública de saúde (Sousa, 2016). 
- O Siclom foi criado com o objetivo do gerenciamento logístico dos medicamentos antirretrovirais. O sistema permite que o usuário se mantenha atualizado em relação ao fornecimento de medicamentos aos pacientes em TARV, nas várias regiões do país (Sousa, 2016).

Foram incluídos no estudo: (i) os casos de AIDS notificados no Sinan entre 2008 e 2018, (ii) os óbitos registrados no SIM e classificados como causa básica 'AIDS', sob os códigos B20 a B24 da Classificação Estatística Internacional de Doenças e Problemas Relacionados à Saúde (CID) entre 2008 e 2018, e (iii) os casos de AIDS com exames laboratoriais no Siscel e que atenderam aos seguintes critérios definidores: adulto apresentando contagem de linfócitos T-CD4+ abaixo de 350 células $/ \mathrm{mm}^{3}$ e carga viral detectável; criança apresentando contagem de linfócitos T-CD4+ menor que a esperada para a idade atual e duas cargas virais maiores que 10 mil cópias $/ \mathrm{mL}$; adulto apresentando contagem de linfócitos T-CD4+ menor que 350 células $/ \mathrm{mm}^{3}$ e uso de medicamento registrado no Siclom; ou criança apresentando contagem de linfócitos T-CD4+ menor que a esperada para a idade atual e uso de medicamento registrado no Siclom (Governo do Distrito Federal, 2010). Além disso, os registros do Siclom foram utilizados para confirmação dos casos de AIDS com testes laboratoriais registrados no Siscel que atenderam aos critérios definidores de AIDS.

\section{Resultados}

Segundo dados do Boletim Epidemiológico HIV/AIDS 2019, no Brasil, no período de 2008 a 2018, foram notificados 275.043 casos de infecção pelo HIV no Sinan, sendo 20.149 casos na região Centro-Oeste (7,32\% do total do país). Em relação ao Distrito Federal, nesse mesmo período, foram notificados 4.316 casos no Sinan (1,56\% em relação ao Brasil e 21,4\% em relação à região Centro-Oeste). No ano de 2018, foram notificados 43.941 casos de infecção pelo HIV no país, sendo 3.625 casos $(8,2 \%)$ na região Centro-Oeste e 634 casos $(1,4 \%)$ no DF. Tais dados estão elencados na Tabela 1:

Tabela 1 - Casos de HIV notificados no Sinan, no Brasil, Centro-Oeste e Distrito Federal por ano de diagnóstico, de 2008 a 2018.

\begin{tabular}{|c|c|c|c|c|c|c|c|c|c|c|c|}
\hline Local & 2008 & 2009 & 2010 & 2011 & 2012 & 2013 & 2014 & 2015 & 2016 & 2017 & 2018 \\
\hline Brasil & 8347 & 9055 & 10693 & 12702 & 14564 & 20459 & 31028 & 38645 & 41166 & 44443 & 43941 \\
\hline \multicolumn{12}{|l|}{ Centro- } \\
\hline Oeste & 294 & 379 & 501 & 795 & 908 & 1455 & 2402 & 3036 & 3120 & 3634 & 3625 \\
\hline \multicolumn{12}{|l|}{ Distrito } \\
\hline Federal & 44 & 58 & 102 & 271 & 337 & 416 & 604 & 604 & 618 & 628 & 634 \\
\hline
\end{tabular}

Fonte: Boletim Epidemiológico HIV/AIDS (2019).

A tabela acima mostra a evolução da notificação de casos de HIV no Sinan em relação ao território federal, à região Centro-Oeste e ao Distrito Federal. Observa-se uma maior quantidade de casos notificados entre 2008 e 2018, denotando um uso ampliado e controle mais rígido dos sistemas de informação governamentais referentes aos agravos de notificação.

O Brasil tem registrado uma média anual de 40.544 novos casos de AIDS entre 2008 e 2018. Verifica-se que o número anual de casos de AIDS no país vem diminuindo desde 2013, quando atingiu 42.934 casos; em 2018, foram registrados 37.161 casos. No período de 2008 a 2018, a região Centro-Oeste apresentou uma média de 3,04 mil casos/ano. O DF, por sua vez, apresentou média de 578 casos/ano. É possível observar que, a partir de 2013, todos apresentaram declínio no número de 
casos de AIDS, sendo que no Brasil o percentual de queda foi de 13,4\%, no CO de 9,3\% e no DF de 36,9\%. É possível observar essa tendência quanto aos casos de AIDS no Brasil, CO e DF na Tabela 2.

A distribuição proporcional dos casos de AIDS mostra uma concentração nas regiões Sudeste e Sul, enquanto as regiões Centro-Oeste, Norte e Nordeste apresentam as menores taxas. Nos últimos anos, entretanto, as regiões Sul, Sudeste e Centro-Oeste apresentaram tendência à queda, enquanto o Norte e Nordeste apresentaram tendência ao crescimento, além de aumento na taxa de mortalidade (Ministério da Saúde, 2019).

Tabela 2 - Casos de AIDS notificados no Sinan, declarados no SIM e registrados no Siscel/Siclom do Brasil, Centro-Oeste e Distrito Federal, de 2008 a 2018.

\begin{tabular}{|c|c|c|c|c|c|c|c|c|c|c|c|}
\hline Local & 2008 & 2009 & 2010 & 2011 & 2012 & 2013 & 2014 & 2015 & 2016 & 2017 & 2018 \\
\hline Brasil & 40857 & 40732 & 40292 & 42273 & 42184 & 42934 & 42122 & 40506 & 38924 & 37999 & 37161 \\
\hline \multicolumn{12}{|l|}{ Centro- } \\
\hline Oeste & 2474 & 2548 & 2574 & 2790 & 2992 & 3072 & 2962 & 2769 & 2700 & 2828 & 2784 \\
\hline \multicolumn{12}{|l|}{ Distrito } \\
\hline Federal & 484 & 509 & 502 & 617 & 607 & 652 & 557 & 516 & 487 & 438 & 411 \\
\hline
\end{tabular}

Fonte: Boletim Epidemiológico HIV/AIDS (2019); Boletim Epidemiológico HIV/AIDS (2018).

É importante observar na Tabela 2 o declínio nos casos de AIDS notificados no Sinan entre 2008 e 2018 nas 3 esferas expostas (Brasil, CO e DF), apesar do aumento do número de casos de HIV notificados na mesma época. Tal dado é importante para a análise da implementação de ações e políticas em saúde, visando a diminuição da patogenicidade e da disseminação da infecção pelo HIV.

Nos últimos anos, a taxa de detecção de AIDS tem caído no Brasil. Em um período de dez anos, a taxa de deteç̧ão apresentou queda de 17,6\%: em 2008, foi de 21,6 casos por 100.000 habitantes e, em 2018, de 17,8 casos a cada 100.000 hab.

A região Centro-Oeste, apesar de ter apresentado menores variações nas taxas anuais, apresentou queda de 4,4\% nos últimos dez anos, de 18,1/100.000 hab. em 2008 para 17,3/100.000 hab. em 2018. Na Tabela 3, entre os anos de 2008 e 2018, observa-se declínio da taxa de detecção de AIDS no DF (25,8\%), a qual passou de 18,6/100.000 hab. em 2008 para 13,8/100.000 hab. em 2018. Esses dados podem ser observados na Tabela 3.

Tabela 3 - Taxa de detecção (por 100.000 hab.) de casos de AIDS notificados no Sinan no Brasil, Centro-Oeste e Distrito Federal, de 2008 a 2018.

\begin{tabular}{lrrrrrrrrrrrr} 
Local & \multicolumn{2}{c}{$\mathbf{2 0 0 8}$} & $\mathbf{2 0 0 9}$ & $\mathbf{2 0 1 0}$ & $\mathbf{2 0 1 1}$ & $\mathbf{2 0 1 2}$ & $\mathbf{2 0 1 3}$ & $\mathbf{2 0 1 4}$ & $\mathbf{2 0 1 5}$ & $\mathbf{2 0 1 6}$ & $\mathbf{2 0 1 7}$ & $\mathbf{2 0 1 8}$ \\
Brasil & 21,6 & 21,3 & 21,2 & 22 & 21,7 & 21,4 & 20,6 & 19,8 & 18,9 & 18,3 & 17,8 \\
Centro- & & & & & & & & & & & \\
Oeste & 18,1 & 18,4 & 18,5 & 19,8 & 20,8 & 20,4 & 19,4 & 17,9 & 17,2 & 17,8 & 17,3 \\
Distrito & & & & & & & & & & & \\
Federal & 18,6 & 19,4 & 19,6 & 23,8 & 22,9 & 23,5 & 19,5 & 17,7 & 16,4 & 14,4 & 13,8
\end{tabular}

Fonte: Boletim Epidemiológico HIV/AIDS (2019). 
A diminuição da taxa de detecção nas 3 esferas apresentadas na Tabela 3 é um dado crucial para compreender o perfil da AIDS na população brasileira e analisar diferenças regionais no enfrentamento e controle do HIV. Levantar hipóteses para explicar um maior declínio percentual no DF em comparação com o restante do CO é importante para corrigir vulnerabilidades na saúde pública da região.

A evolução dos óbitos por causa básica AIDS no Brasil, Centro-Oeste e Distrito Federal pode ser observada na Tabela 4. Entre 2008 e 2018, constatam-se no Brasil 133.337 óbitos tendo o HIV/AIDS como causa básica. A região Centro-Oeste é responsável por $8.372(6,2 \%)$ desse total. Ainda nessa perspectiva, o DF apresenta 1.262 (15,07\% em relação ao CO) desses casos, sendo que o ano de 2008 apresentou 105 óbitos por causa básica AIDS e o ano de 2018, 107 óbitos.

Tabela 4 - Óbitos por causa básica AIDS no Brasil, Centro-Oeste e Distrito Federal, de 2008 a 2018.

$\begin{array}{lcccccccccccc}\text { Local } & \mathbf{2 0 0 8} & \mathbf{2 0 0 9} & \mathbf{2 0 1 0} & \mathbf{2 0 1 1} & \mathbf{2 0 1 2} & \mathbf{2 0 1 3} & \mathbf{2 0 1 4} & \mathbf{2 0 1 5} & \mathbf{2 0 1 6} & \mathbf{2 0 1 7} & \mathbf{2 0 1 8} & \mathbf{2 0 0 8 - 2 0 1 8} \\ \text { Brasil } & 11839 & 12134 & 12151 & 12151 & 12073 & 12564 & 12575 & 12667 & 12540 & 11663 & 10980 & 133337 \\ \begin{array}{l}\text { Centro- } \\ \text { Oeste }\end{array} & 725 & 705 & 774 & 711 & 772 & 734 & 746 & 831 & 818 & 804 & 752 & 8372 \\ \begin{array}{l}\text { Distrito } \\ \text { Federal }\end{array} & 105 & 116 & 118 & 117 & 112 & 126 & 128 & 114 & 112 & 107 & 107 & 1262\end{array}$

Fonte: Boletim Epidemiológico HIV/AIDS (2019).

É importante observar na Tabela 4 a participação do DF no quantitativo de óbitos por AIDS no Brasil e CentroOeste. A proporção de $15,07 \%$ de óbitos no DF em relação ao CO levanta questionamentos a respeito da rede de saúde na capital, especialmente ao questionar se a disponibilidade de recursos hospitalares e a procura de assistência médica no Distrito Federal seriam fatores importantes para a mortalidade no local.

A Tabela 5 traz um dado importante para a análise da situação epidemiológica do HIV/AIDS: o coeficiente de mortalidade bruto.

Tabela 5 - Coeficiente de mortalidade bruto por AIDS (por 100.000 hab.) no Brasil, Centro-Oeste e Distrito Federal por ano de óbito, de 2008 a 2018.

$\begin{array}{lccccccccccc}\text { Local } & \mathbf{2 0 0 8} & \mathbf{2 0 0 9} & \mathbf{2 0 1 0} & \mathbf{2 0 1 1} & \mathbf{2 0 1 2} & \mathbf{2 0 1 3} & \mathbf{2 0 1 4} & \mathbf{2 0 1 5} & \mathbf{2 0 1 6} & \mathbf{2 0 1 7} & \mathbf{2 0 1 8} \\ \text { Brasil } & 6,2 & 6,3 & 6,4 & 6,3 & 6,2 & 6,2 & 6,2 & 6,2 & 6,1 & 5,6 & 5,3 \\ \begin{array}{l}\text { Centro- } \\ \text { Oeste }\end{array} & 5,3 & 5,1 & 5,5 & 5 & 5,4 & 4,9 & 4,9 & 5,4 & 5,2 & 5,1 & 4,7 \\ \text { Distrito } & & & & & & & & & & & \\ \text { Federal } & 4,7 & 4,4 & 4,6 & 4,5 & 4,2 & 4,5 & 4,5 & 3,9 & 3,8 & 3,5 & 3,6\end{array}$

Fonte: Boletim Epidemiológico HIV/AIDS (2019).

Na Tabela 5 está explícita a queda no coeficiente de mortalidade bruto do Brasil, que passou de 6,2\% para 5,3\% óbitos por 100.000 hab. Ademais, no Centro-Oeste, também se registrou diminuição do coeficiente de mortalidade bruto, de 5,3\% para 4,7\% óbitos/100.000 hab. Em todo o período, o DF apresentou coeficiente de mortalidade bruto inferior ao nacional, 
sendo de 4,7\% em 2008, exibindo em 2018 a menor taxa, com 3,6 óbitos/100.000 hab. É imprescindível observar tais diferenças regionais para analisar diferenças na assistência à saúde da população brasileira.

\section{Discussão}

A infecção pelo HIV e a AIDS representam fenômeno global, dinâmico e instável, cuja forma de ocorrência nas diferentes regiões do mundo depende, entre outros determinantes, do comportamento humano individual e coletivo. No Brasil, o agravo apresenta caráter complexo e se configura como verdadeiro mosaico de subepidemias regionais (Brito, 2001). Uma ferramenta muito importante no combate ao HIV é a monitorização de dados epidemiológicos, de forma a identificar alterações no padrão de morbimortalidade da doença ao longo dos anos.

O país tem registrado, anualmente, uma média de 39 mil novos casos de AIDS nos últimos cinco anos. Apesar disso, desde o ano de 2012, observa-se uma diminuição na taxa de deteç̧ão de AIDS no Brasil, como resultado direto da recomendação do "tratamento para todos", implementada em dezembro de 2013 como estratégia governamental em saúde. Essa estratégia tem como objetivo disponibilizar as medicações antirretrovirais nas unidades de saúde para todos os pacientes, independentemente dos exames de CD4 e da carga viral. Da mesma forma, houve importante decréscimo do coeficiente de mortalidade - também, possivelmente, como consequência da recomendação do "tratamento para todos" e da ampliação do diagnóstico precoce da infecção pelo HIV (Ministério da Saúde, 2018).

Entretanto, a infecção pelo HIV no Brasil sofreu aumento a partir de 2013, e isso se deve, também, ao fato de que a notificação compulsória da infecção pelo HIV teve início somente em 2014, dificultando uma análise epidemiológica mais rigorosa com relação à real tendência do perfil da infecção no Brasil.

Outro fator importante relacionado à morbimortalidade do HIV/AIDS é a prevenção combinada, que consiste na conjugação de abordagens possíveis de estratégias de prevenção para o HIV, sendo elas (1) a abordagem biomédica, (2) a abordagem comportamental e (3) a abordagem estrutural. Essas ações são centradas nos indivíduos, em seus grupos sociais e na sociedade em que vivem, mediante ações que levem em consideração as necessidades, especificidades e as formas de transmissão do vírus (Ministério da Saúde, 2017).

Intervenções biomédicas são estratégias voltadas à redução do risco de exposição ou de transmissibilidade. Como exemplos, tem-se a distribuição de preservativos masculinos e femininos e a introdução do Tratamento para Todas as Pessoas (TTP); a Profilaxia Pós-Exposição (PEP); e a Profilaxia Pré-Exposição (PrEP). (Ministério da Saúde, 2017).

As intervenções comportamentais são estratégias que contribuem para o aumento da informação e conhecimento e para a percepção ou autoavaliação do risco à exposição ao HIV, contribuindo para a redução desse risco, mediante incentivos a mudanças de comportamento individual ou comunitário. Como exemplos, podem ser citados: incentivo ao uso de preservativos masculinos e femininos; aconselhamento sobre HIV/AIDS e outras IST; incentivo à testagem; adesão às intervenções biomédicas; vinculação e retenção nos serviços de saúde; redução de danos para as pessoas que usam álcool e outras drogas; e estratégias de comunicação e educação entre pares (Ministério da Saúde, 2017).

Por fim, as intervenções estruturais são estratégias voltadas a enfrentar fatores e condições socioculturais que influenciam diretamente a vulnerabilidade de indivíduos ou grupos sociais específicos que sofrem preconceito, estigma, discriminação, violência ou qualquer outra forma de alienação dos direitos e garantias fundamentais à dignidade humana, tais como ações de enfrentamento ao racismo, sexismo, LGBTfobia e demais preconceitos; promoção e defesa dos direitos humanos; campanhas educativas e de conscientização (Ministério da Saúde, 2017).

Ao analisar o DF e compará-lo com a região Centro-Oeste e ao Brasil, em um período de dez anos, é possível ter um panorama do controle da disseminação da enfermidade e da sua participação no perfil de doenças infectocontagiosas da unidade federativa. O DF apresentou média inferior ao Brasil e superior ao Centro-Oeste em relação à taxa de detecção de 
AIDS. Em relação ao coeficiente de mortalidade, o DF mostrou média inferior ao Centro-Oeste e ao Brasil. Com relação aos casos de HIV, entre 2008 e 2018, o DF contabilizou 21,4\% dos casos em relação ao Centro-Oeste. Ademais, em 2011, 2012 e 2013 o DF apresentou taxa de detecção de AIDS superior à taxa nacional.

\section{Conclusão}

A partir dos resultados e de forma congruente à literatura, durante o período estudado, observa-se queda do número de casos de AIDS, da taxa de detecção de AIDS, dos óbitos e do coeficiente de mortalidade no Brasil, no Centro-Oeste e no Distrito Federal, devido à disponibilização do tratamento pelo serviço público de saúde. No entanto, mais recentemente, verifica-se aumento do número de casos de HIV, a considerar que a notificação compulsória do HIV data de 2014. Nesse contexto, os dados epidemiológicos revelam a importância da consideração do HIV/AIDS como problema de saúde pública, havendo a necessidade da realização das ações preventivas e educativas de promoção à saúde sexual e de prevenção, além da ampliação, principalmente na atenção primária em saúde, da oferta do diagnóstico precoce e do tratamento oportuno e adequado (Pereira, 2007).

Como sugestões para trabalhos futuros, cita-se conduzir estudos semelhantes ao deste trabalho, a fim de melhor entendimento da situação da AIDS no Brasil e em suas regiões, auxiliando na implementação e condução de políticas públicas voltadas para a população alvo.

\section{Referências}

Barros, S. G. \& Vieira-da-Silva, L.M. (2016). The genesis of the AIDS policy and AIDS Space in Brazil (1981-1989). Revista de Saúde Pública. 50, 43.

Brito, A. M., Castilho, E. A. \& Szwarcwald, C. L. (2001). AIDS e infecção pelo HIV no Brasil: uma epidemia multifacetada. Rev. Soc. Bras. Med. Trop. $34(2), 207-17$.

Greco, D. B. (2016). Trinta anos de enfrentamento à epidemia da Aids no Brasil, 1985-2015. Ciênc. saúde coletiva. 21(5), 1553-64.

Guimarães, M. D., Carneiro, M., Abreu, D. M. \& França, E. B (2017). Mortalidade por HIV/AIDS no Brasil, 2000-2015: motivos para preocupação? Revista Brasileira de Epidemiologia. 20(1); 182-190.

Laguardia, J., Domingues, C. M., Carvalho, C., Lauerman, C. R., Macário, E., \& Glatt, R. (2004). Sistema de informação de agravos de notificação em saúde (Sinan): desafios no desenvolvimento de um sistema de informação em saúde. Epidemiologia e Serviços de Saúde. 13(3); 135-146.

Levy, J. A., Autran, B., Coutinho, R. A., \& Phair, J. P. (2012). 25 Years of AIDS: recording progress and future challenges. AIDS. 26(10):1187-9.

Ministério da Saúde (2020). Departamento de Condições Crônicas e Infecções Sexualmente Transmissíveis: História da AIDS.

Ministério da Saúde (2019). Departamento de IST, HIV/AIDS e Hepatites Virais: Boletim Epidemiológico AIDS e DST.

Ministério da Saúde (2018). Departamento de IST, HIV/AIDS e Hepatites Virais: Boletim Epidemiológico AIDS e DST.

Ministério da Saúde (2017). Departamento de Vigilância, Prevenção e Controle das Infecções Sexualmente Transmissíveis, do HIV/AIDS e das Hepatites Virais: Prevenção Combinada do HIV. Brasília.

Ministério da Saúde (2017). Departamento de doenças de condições crônicas e infecções sexualmente transmissíveis: O que é a prevenção combinada.

Morais, R. M. \& Costa, A. L. (2017). Uma avaliação do Sistema de Informações sobre Mortalidade. Saúde em Debate. 41 ; $101-117$.

Pereira, C. C., Machado, C. J. \& Rodrigues, R. N. (2007). Perfis de causas múltiplas de morte relacionadas ao HIV. AIDS nos municípios de São Paulo e Santos, Brasil, 2001. Cad. Saúde Pública. 23(8); 645-655.

Pereira, G. F., Shimizu, H. E., Bermudez, X. P. \& Hamann, E. M. (2018). Epidemiologia do HIV e AIDS no estado do Rio Grande do Sul, $1980-2015$. Epidemiologia e Serviços de Saúde. 27(4); e2017374.

Governo do Distrito Federal (2010). Estabelece os documentos que deverão ser fornecidos pelo médico assistente ao paciente para fins de cadastramento nas unidades de saúde dispensadoras de medicamentos antirretrovirais. Portaria n .37 de 23 de março de 2010, Diário Oficial do Distrito Federal. N ${ }^{\circ} 58$, p.10.

Reis, A. C., Santos, E. M., \& Cruz, M. M. (2007). A mortalidade por AIDS no Brasil: um estudo exploratório de sua evolução temporal. Epidemiologia e Serviços de Saúde. 16(10): 195-205.

Sousa, A. I. \& Pinto Júnior, V. L. (2016). Análise espacial e temporal dos casos de AIDS no Brasil em 1996-2011: áreas de risco aumentado ao longo do tempo. Epidemiologia e Serviços de Saúde. 25(3); 467-476. 
Research, Society and Development, v. 10, n. 5, e58010515312, 2021

(CC BY 4.0) | ISSN 2525-3409 | DOI: http://dx.doi.org/10.33448/rsd-v10i5.15312

UNAIDS (2016). Global AIDS Response Progress Reporting (GARPR). UNAIDS 2016 estimates.

Vella, S., Schwartländer, B., Sow, S. P., Eholie, S. P. \& Murphy, R. L. (2012). The history of antiretroviral therapy and of its implementation in resourcelimited areas of the world. AIDS. 26(10): 1231-1241.

Koche, J. (2015). Fundamentos de metodologia científica: teoria da ciência e iniciação à pesquisa. (34a ed.), Vozes. 